\title{
Chern-Simons number diffusion in (1+1)-dimensional Higgs theory
}

\author{
Ph. de Forcrand and A. Krasnitz * \\ Interdisziplinäres Projektzentrum für Supercomputing \\ Eidgenössische Technische Hochschule \\ CH-8092 Zürich, Switzerland \\ R. Potting \\ Universidade do Algarve \\ Unidade de Ciências Exactas e Humanas \\ Campus de Gambelas, 8000 Faro, Portugal
}

July 1994

\begin{abstract}
We study the Chern-Simons number diffusion rate in the $(1+1)$-dimensional lattice Abelian Higgs model at temperatures much higher than, as well as comparable to, the sphaleron energy. It is found that in the high-temperature limit the rate is likely to grow as power $2 / 3$ of the temperature. In the intermediatetemperature regime, our numerical simulations show that very weak temperature dependence of the rate, found in previous work, persists at smaller lattice spacings. We discuss possibilities of relating the observed behavior of the rate to static finite-temperature properties of the model.
\end{abstract}

*Address after 1 September 1994: Niels Bohr Institute, Blegdamsvej 17, DK-2100 Copenhagen, Denmark. 
The Abelian Higgs model (AHM) in $1+1$ dimensions is one of the simplest yet nontrivial field-theoretic analogs of the Standard Model (SM) as far as fermion number nonconservation at high temperature is concerned. Clearly, there is little hope of quantitatively understanding this phenomenon in SM if a similar goal cannot be achieved for AHM, which lends itself much more easily to both analytical and numerical studies. This explains the considerable amount of work done on this aspect of AHM in recent

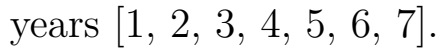

Prior to the present work, the status of knowledge on thermally induced fermionnumber violation in AHM has been as follows. Much like in SM, the dynamics of the Chern-Simons number $N_{\mathrm{CS}}$ (and thus of the fermion number) in AHM can reliably be studied in the classical approximation at temperatures well above the gauge- $\left(m_{W}\right)$ and Higgs- $\left(m_{H}\right)$ boson mass scales. The relative simplicity of AHM allows to give an accurate estimate of the $N_{\mathrm{CS}}$ diffusion rate at temperatures $T$ low compared to the energy $E_{\mathrm{sph}}$ of a sphaleron saddle-point configuration separating vacua labeled by consecutive integer values of $N_{\mathrm{CS}}$. Not surprisingly, the rate $\Gamma$ exhibits thermal-activation behavior [2]

$$
\Gamma=f(\xi) T^{2 / 3}\left(\frac{E_{\mathrm{sph}}}{T}\right)^{\frac{7}{6}} \exp \left(-\frac{E_{\mathrm{sph}}}{T}\right), \quad m_{W}, m_{H} \ll T \ll E_{\mathrm{sph}},
$$

where $\xi=2 m_{W}^{2} / m_{H}^{2}$. Unlike the SM case, in AHM both the sphaleron energy and the $f$ prefactor are known analytically. Numerical real-time simulations of AHM at low temperatures, including those of the current work, indeed agree with (1) [1, 田, 5].

Surprising as it may sound, at the opposite end of thermal scale, $T \gg E_{\mathrm{sph}}$, less was known about the rate in AHM than in SM. In SM, the only dimensionful quantity relevant at such high temperatures is the temperature itself, hence, on dimensional grounds, the rate is expected to behave as $T^{4}$. In AHM, however, both the gauge and the quartic Higss coupling constants are dimensionful, and therefore, while a power-law behavior was generally believed to be satisfied [8, 9], the corresponding power could not be established from dimensional considerations. Nevertheless, a simple scaling argument given in the following indicates that this power is $2 / 3$. This power law is also consistent with our numerical results.

As usually is the case, the intermediate-temperature regime ( $T$ comparable to $\left.E_{\mathrm{sph}}\right)$ is the least accessible to analytical estimates. Numerical work [4, 旬 revealed a surprising feature: at temperatures 5 to 7 times less than $E_{\mathrm{sph}}$ the rate practically stops growing and remains roughly constant until the sphaleron scale of temperatures is reached. Our simulations show that this phenomenon persists at smaller lattice spacings and is therefore not a lattice artifact.

We shall now elaborate on the points just made, discussing first the physics and afterwards explaining our numerical methods. We begin by giving a scaling argument in favor of the $T^{2 / 3}$ behavior of the rate at high temperatures. Consider the latticeregularized classical AHM whose temporal-gauge Hamiltonian can, with a suitable choice 
of units, be written in a dimensionless form [3, 田:

$$
H(v, a)=\frac{1}{2 a} \sum_{j=1}^{N}\left[\xi E_{j, j+1}^{2}+\left|\pi_{j}\right|^{2}+\frac{a^{2}}{2}\left(\left|\phi_{j}\right|^{2}-v^{2}\right)^{2}+\left|\phi_{j+1}-e^{i a A_{j, j+1}} \phi_{j}\right|^{2}\right],
$$

where $\pi_{j}$ and $E_{j, j+1}$ are canonically conjugate momenta of the complex scalar (Higgs) field $\phi_{j}$ and the spatial component $A_{j, j+1}$ of the gauge field, residing respectively on sites and links of an $N$-site chain. The lattice spacing is $a$. Periodic boundary conditions are assumed. The reader can verify by direct substitution that if $\phi_{j}(t), \pi_{j}(t), A_{j, j+1}(t)$, $E_{j, j+1}(t)$ are solutions at time $t$ of the equations of motion following from $H(v, a)$ with $H(v, a)=\mathcal{E}$, then $c \phi_{j}(c t), c \pi_{j}(c t), c A_{j, j+1}(c t), c E_{j, j+1}(c t)$ play the same role at time $t$ for $H(c v, a / c)$ with $H(c v, a / c)=c^{3} \mathcal{E}$. Because of this property, scaling laws can be derived for both static and dynamical observables in AHM. Consider in particular the Chern-Simons number defined as

$$
N_{\mathrm{CS}}(t)=\frac{a}{2 \pi} \sum_{j=1}^{N} A_{j, j+1}(t)
$$

Its diffusion rate is

$$
\Gamma(a, v, T)=\lim _{N, t \rightarrow \infty} \frac{1}{a N t}\left\langle\left(N_{\mathrm{CS}}(t)-N_{\mathrm{CS}}(0)\right)^{2}\right\rangle_{T} .
$$

By \langle\rangle$_{T}$ we mean integration over all the initial values of dynamical variables [10 with the Boltzmann weight

$$
\exp \left(-\frac{H(v, a)}{T}\right) \prod_{j=1}^{N} \delta\left(C_{j}(a)\right)
$$

where the delta-function factors impose a set of Gauss' constraints

$$
C_{j}(a) \equiv \frac{1}{a}\left(E_{j, j+1}-E_{j-1, j}\right)-\operatorname{Im}\left(\pi_{j} \phi_{j}\right)=0 .
$$

Multiplying all the integration variables (i.e., all the initial values of fields and momenta) in (四) by $c$ and using the scaling property of $H(v, a)$ under this transformation we obtain a simple scaling rule for the rate:

$$
\Gamma\left(\frac{a}{c}, c v, c^{3} T\right)=c^{2} \Gamma(a, v, T) .
$$

Differentiating with respect to $c$ at $c=1$ gives a differential equation for $\Gamma$ as a function of $a, v, T$ :

$$
\left(v \partial_{v}+3 T \partial_{T}-a \partial_{a}-2\right) \Gamma=0
$$

whose most general solution is

$$
\Gamma=T^{\frac{2}{3}} g\left(a^{3} T, \frac{v^{3}}{T}\right)
$$




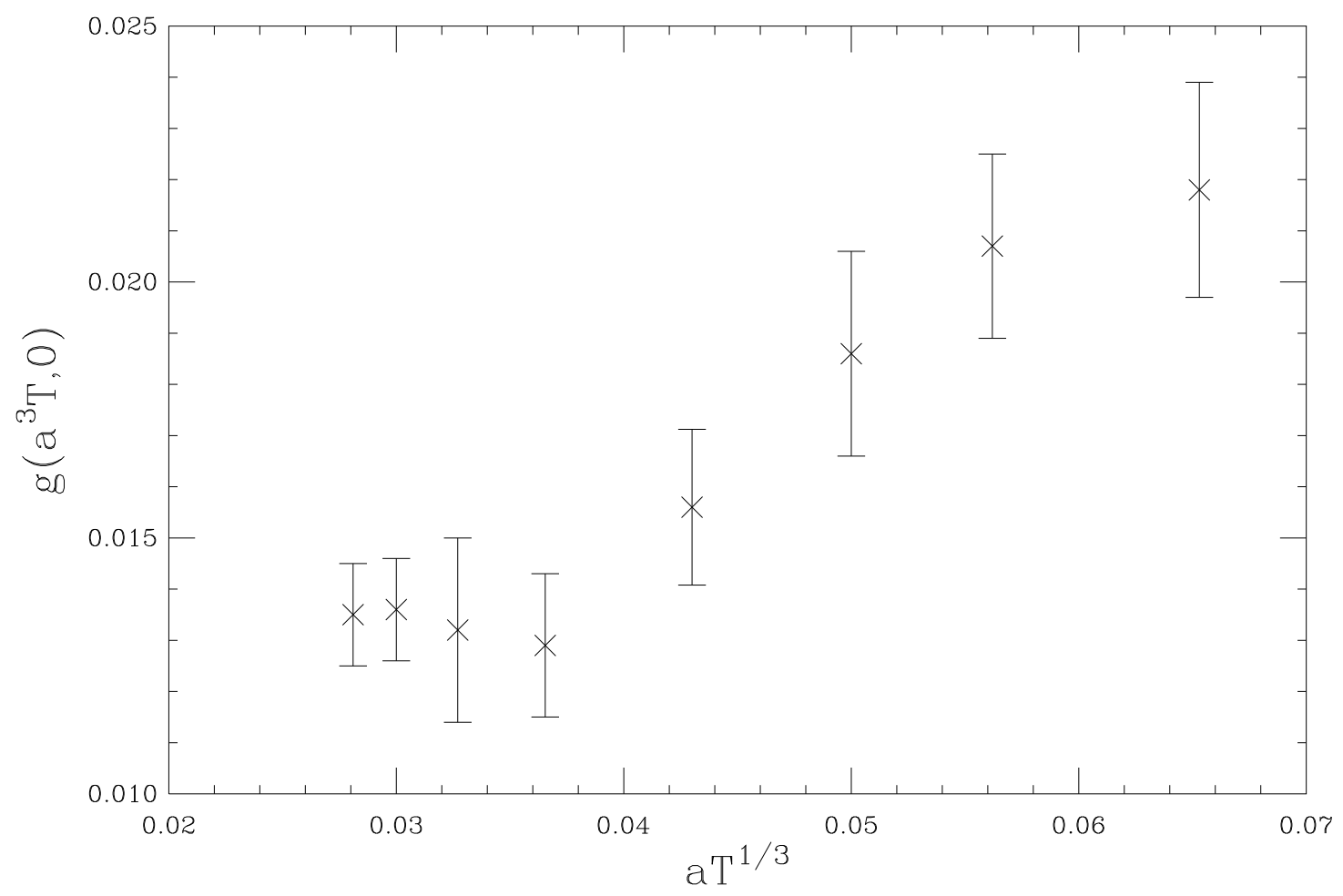

Figure 1: $g\left(a^{3} T, 0\right)$ measured at small values of $a T^{1 / 3}$.

where $g(x, y)$ is an arbitrary function of its two arguments. In particular, since $E_{\mathrm{sph}} \propto v^{3}$, (四), derived for the continuum theory, shows that $g(0, y) \propto y^{7 / 6} \exp (-y)$ for $y \gg 1$. As for the temperatures far above the sphaleron scale, the continuum limit of the rate, if it exists, is $g(0,0) T^{2 / 3}$ as claimed. Both result (1) and numerical evidence strongly suggest that the rate does indeed have a finite continuum limit for any nonzero $v^{3} / T$. It is also plausible that the rate becomes insensitive to the details of the Higgs potential at energy scales far below $T$, i.e., $\Gamma$ becomes independent of $v$ if $v^{3} \ll T$. We therefore expect $0<g(0,0)<\infty$.

We verified the scaling rule (7) by a numerical simulation. Since in this part of the work we are primarily interested in the high-temperature regime, we simplify matters by setting $v=0$. The rate measurement then gives

$$
\frac{\Gamma(a=1 / 2, T=1)}{\Gamma(a=1, T=1 / 8)}=4 . \pm 0.4
$$

in agreement with (7). Next, we performed a series of rate measurements closer to the continuum limit of the $v=0$ theory in order to estimate $g(0,0)$. The measurements, done at lattice spacings $1 / 16$ and $1 / 8$, span a range of values of the scaling-invariant parameter $a T^{1 / 3}$ between 0.028 and 0.065 . The results, presented in Figure 1, indicate that $g(x, 0)$ approaches a finite value as $x \rightarrow 0$.

In this work we are concerned with classical AHM. Note, however, that if the classical approximation for the rate is valid in quantum AHM at very high temperatures, so is 


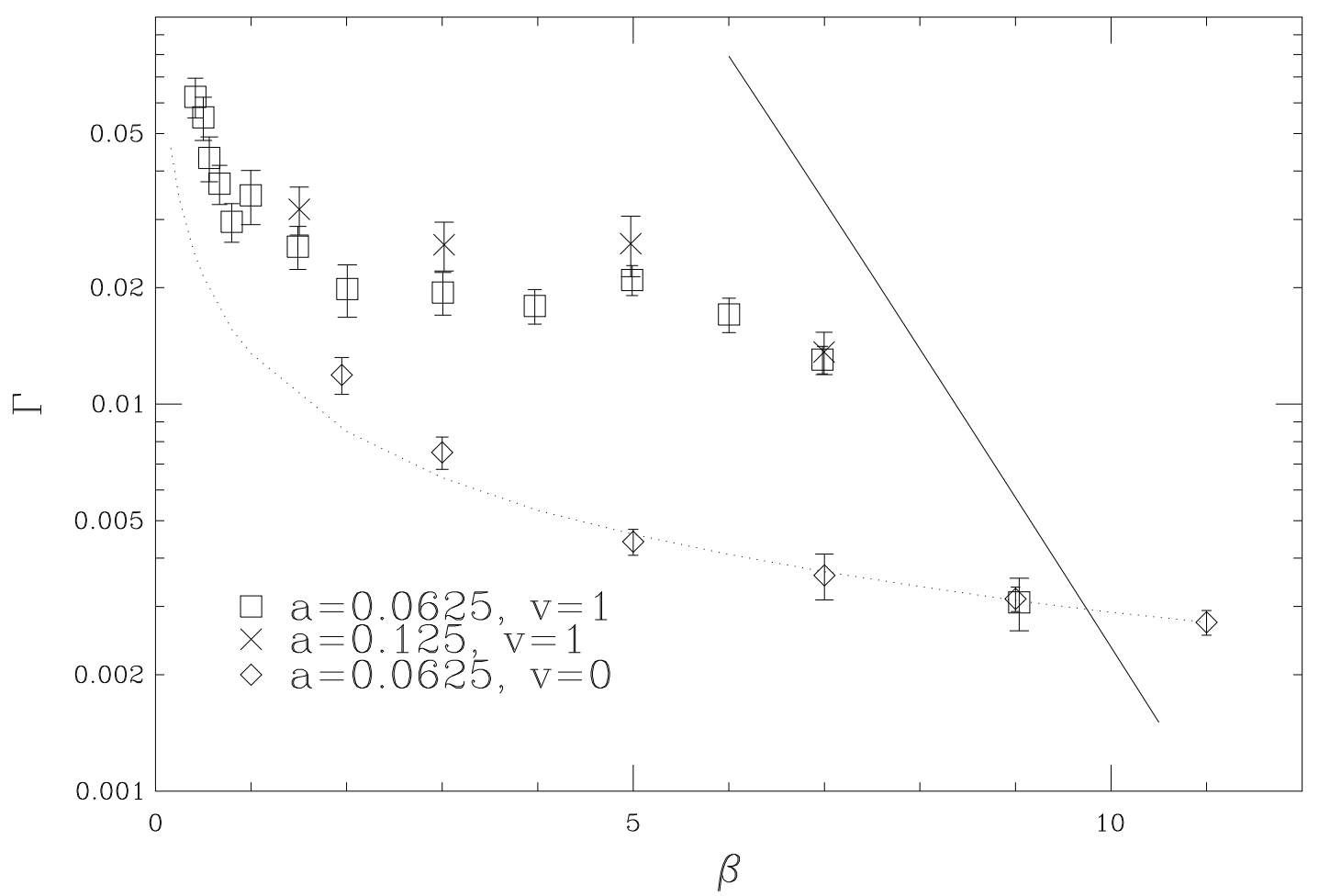

Figure 2: Measured rate dependence on the inverse temperature $\beta$. The solid line is the activation-theory prediction. The dashed line shows the $T^{2 / 3}$ law for the hightemperature theory, with $g(0,0)$ estimated at $\beta=11, a=1 / 16$.

the $T^{2 / 3}$ law. Indeed, for consistency of the classical approximation the classical lattice spacing is chosen inversely proportional to the temperature [1, [5], yielding $a^{3} T \rightarrow 0$, as required in order for the $T^{2 / 3}$ law to hold.

Next, we studied the intermediate-temperature properties of the rate. As in previous work on the subject, we fixed $v=1$, resulting in $E_{\mathrm{sph}} \approx 0.94$. The inverse-temperature range of interest is then $1<\beta<5$, where an extremely weak temperature dependence of the rate was found in previous work $[4,5$. By simulating the theory closer to continuum limit ( $a=1 / 16$ compared to $a \geq 1 / 8$ in the earlier work), we verified that this feature is not a lattice artifact: as Figure 2 2 shows, the plateau region becomes wider and more pronounced as the lattice spacing shrinks. At still lower temperatures, $\beta \geq 7$, the lattice-spacing dependence of the rate rapidly diminishes. We find a good agreement between our low-temperature rate measurements and those of [4], performed at larger lattice spacings. Both earlier and current results approach the asymptotic form (四) with decreasing temperature.

Let us summarize the properties of $N_{\mathrm{CS}}$ diffusion rate in AHM as they emerge from this and previous works. At low $\left(T \ll E_{\mathrm{sph}}\right)$ the rate follows the activation-theory predic-

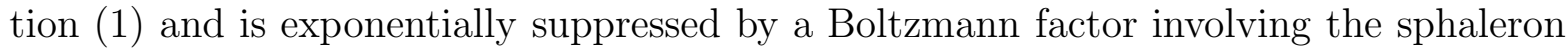
barrier height. At extremely high temperatures $\left(T \gg E_{\mathrm{sph}}\right)$ the rate, if it has a finite con- 


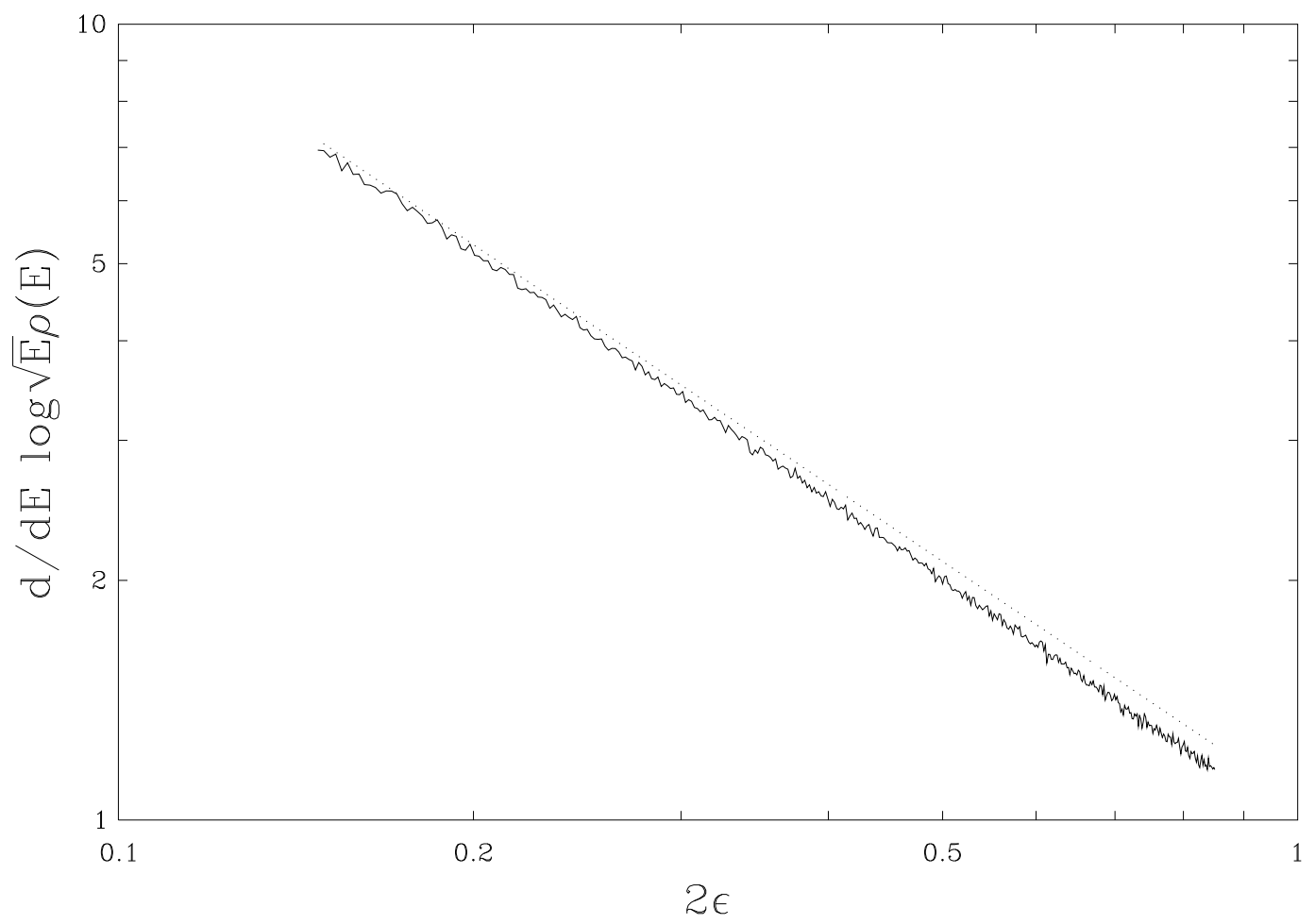

Figure 3: Relative variation of the density of states with energy per degree of freedom in AHM (solid line) and in a free-field theory (dashed line).

tinuum limit, should grow as $T^{2 / 3}$. Our simulations do point to a finite continuum limit of the high-temperature rate. The crossover between the two extreme regimes involves a temperature interval characterized by a very slow or null rate growth. A complete theory of $N_{\mathrm{CS}}$ diffusion should contain a derivation of the $T^{2 / 3}$ law at high temperatures and a determination of $g(0,0)$ from first principles. It also should explain how corrections to either high- or low-temperature rate lead to the observed interpolation between the two extremes. In the absence of such a theory the best we can do is look for clues that might help construct one.

To this end, we examined a possibility that the reasons for the rate suppression

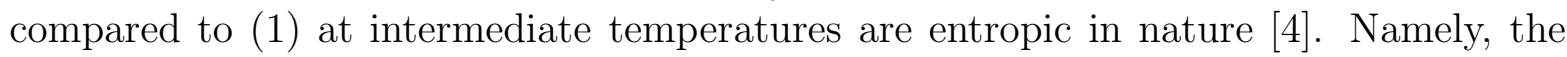
derivation of (1) is based on approximating the AHM phase space by that of linear excitations about the ground state. Starting at very low temperatures $T$, where it is definitely valid, this approximation will only hold with increasing $T$ as long as the freefield relation

$$
\frac{d}{d E(T)} \log (\sqrt{E(T)} \rho(E(T)))=\frac{1}{2 \epsilon(T)}=\frac{1}{T}
$$

approximately holds. Here $E(T)$ is the average energy of the system at temperature $T$, $\epsilon(T)$ is the average energy per degree of freedom, and $\rho(E)$ is the corresponding density of states. We determined the left-hand side of (11) using the binning method [11. As Figure 3 shows, the variation of the AHM density of states hardly differs from, and 


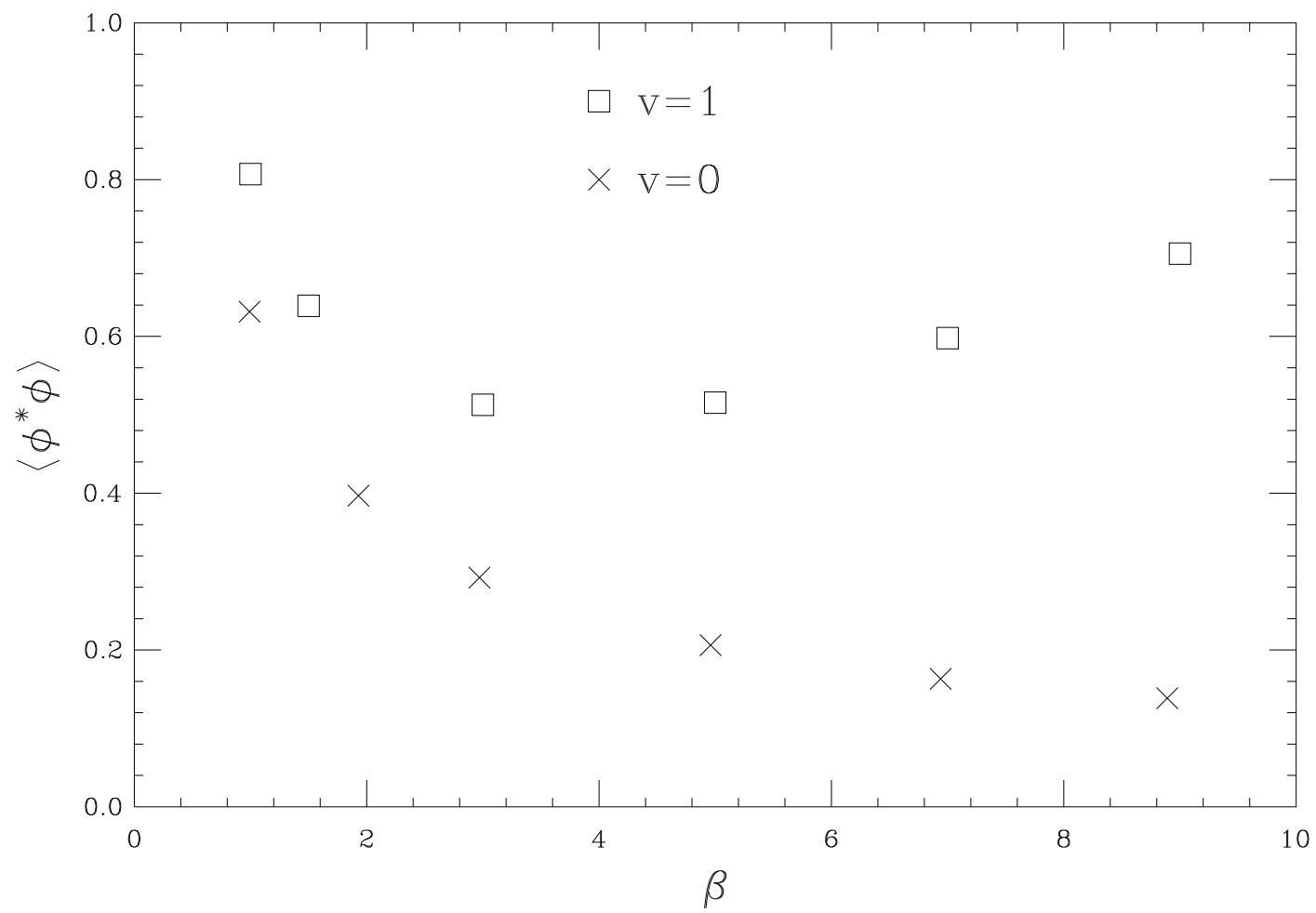

Figure 4: Temperature dependence of $\left\langle|\phi|^{2}\right\rangle_{T}$ in $v=1$ (squares) and $v=0$ (crosses) theories.

even appears somewhat smaller than, that of a free-field theory, even as the energy per degree of freedom (approximately equal to $T / 2$ throughout the range of temperatures in question) approaches the sphaleron scale. We therefore conclude that the observed slowdown of the rate growth is unlikely to be entropy-driven.

In search for other clues we observed that in the intermediate range of temperatures (a) the average squared expectation value $\left\langle\phi^{2}\right\rangle_{T}$ of the scalar field is close to a minimum (Figures 2 and (4); (b) the rate in the theory with $v^{2}=0$ is considerably below that of $v^{2}=1$ theory, while $(c)\left\langle|\phi|^{2}\right\rangle_{T}$ is considerably greater in the $v^{2}=1$ theory than in the $v^{2}=0$ one. Put together, these pieces of empirical evidence suggest that the rate might be closely related to $\left\langle|\phi|^{2}\right\rangle_{T}$. Moreover, repeating our scaling argument for $\left\langle|\phi|^{2}\right\rangle_{T}$, we obtain an analog of (9):

$$
\left\langle|\phi|^{2}\right\rangle_{T}=T^{\frac{2}{3}} h\left(a^{3} T, \frac{v^{3}}{T}\right),
$$

where, again, we expect $0<h(0,0)<\infty$. As our measurements (Table 囵) show, $h\left(a^{3} T, 0\right)=\left\langle|\phi|^{2}\right\rangle_{T} / T^{\frac{2}{3}}$ varies by only about $5 \%$ for $a^{3} T$ between $1.9 \times 10^{-5}$ and $1.25 \times$ $10^{-4}$. We conclude that, in all likelihood, $\left\langle|\phi|^{2}\right\rangle_{T} \propto T^{2 / 3}$ in the continuum limit of high-temperature AHM. As a consequence, we also expect $\Gamma \propto\left\langle|\phi|^{2}\right\rangle_{T}$ in this limit. It therefore appears worthwhile to explore the possibility that at intermediate and high temperatures the time scale for $N_{\mathrm{CS}}$ diffusion is primarily set by the value of $\left\langle|\phi|^{2}\right\rangle_{T}$. 


\begin{tabular}{|c|rrrrrrr|}
\hline$a^{3} T \times 10^{5}$ & 12.6 & 8.20 & 4.93 & 3.52 & 2.73 & 2.24 & 1.89 \\
\hline$\left\langle|\phi|^{2}\right\rangle_{T}$ & 0.615 & 0.605 & 0.600 & 0.594 & 0.595 & 0.599 & 0.588 \\
& \pm 0.005 & \pm 0.004 & \pm 0.004 & \pm 0.004 & \pm 0.007 & \pm 0.004 & \pm 0.004 \\
\hline
\end{tabular}

Table 1: Average squared magnitude of the scalar field as a function of $a^{3} T$ in the $v=0$ theory.

This seems plausible given the equation of motion for the Chern-Simons number written in the form

$$
\ddot{N}_{\mathrm{CS}}=\frac{\xi}{2 \pi} \sum_{j} \rho_{j} \rho_{j+1} \sin b_{j, j+1}=\frac{\xi}{2 \pi}\left\langle|\phi|^{2}\right\rangle_{T} \sum_{j} \sin b_{j, j+1}+\text { corrections }
$$

where $\alpha_{j} \equiv \arg \phi_{j}, \phi_{j}=\rho_{j} \exp \left(i \alpha_{j}\right)$, and $b_{j}=\alpha_{j+1}-\alpha_{j}-a A j, j+1$.

We conclude by explaining simulation methods used in this work. We chose to determine $\Gamma$ as given by (4) by generating a thermal ensemble of initial conditions for lattice AHM. We then let each of these initial field configurations evolve according to the AHM

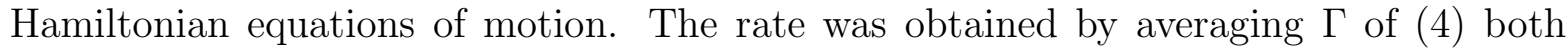
over the ensemble of initial conditions and within individual Hamiltonian trajectories of the system [5]. Initial configurations were generated by applying standard Monte-Carlo techniques to the theory written in terms of gauge-invariant variables [4]:

$$
\begin{aligned}
H^{\prime}= & \frac{a}{2} \sum_{j}\left(\xi \epsilon_{j, j+1}^{2}+\left(\frac{\epsilon_{j, j+1}-\epsilon_{j-1, j}}{a \rho_{j}}\right)^{2}+\left(\frac{\pi_{j}^{\rho}}{a}\right)^{2}+\frac{2}{a^{2}}\left(\rho_{j}^{2}-\rho_{j} \rho_{j+1} \cos b_{j, j+1}\right)\right) \\
& +\frac{a}{4} \sum_{j}\left(\rho_{j}^{2}-1\right)^{2},
\end{aligned}
$$

where $\rho_{j}$ and $b_{j, j+1}$ are defined as in (13), $\epsilon_{j, j+1}=E_{j, j+1} / a$, and $\pi_{j}^{\rho}$ is the canonical momentum of $\rho_{j}$. More specifically, we used the Metropolis algorithm to update the $\rho$ and $b$ variables and the heat-bath algorithm to update $\epsilon$. The fourth canonical variable, $\pi_{\rho}$, is normally distributed and required no iterative procedure to be generated. Note that by choosing to work with gauge-invariant variables we ensured automatic fulfillment of Gauss' law by initial configurations. Having generated an initial configuration, we performed transformation to a gauge-dependent set of variables as in (2) under an initial gauge condition $\arg \phi_{j}=0, A_{j, j+1}=-b_{j, j+1} / a$ and integrated the Hamiltonian equations of motion in terms of this latter variable set. We used the fourth-order leapfrog algorithm [12]. In case of AHM and other gauge theories leapfrog algorithms offer, beside their superior energy-conserving properties, the extra benefit of exact local charge conservation. For the smallest lattice spacing used, $a=1 / 16$, we varied the time step from 0.02 at low temperatures to 0.005 at high temperatures, keeping it small enough to suppress any time-step dependence of observables. As a rule, we set the physical size $\mathrm{Na}$ of the system to 50, known to be large enough to avoid noticeable finite-size effects on the rate and other measured observables [4]. Finally, the value of $\xi$ was 10 throughout our simulations. 


\section{Acknowledgments}

We thank A. Bochkarev, A. Kovner, J. Smit, A. Wipf, W.H. Tang, E. Mottola, S. Habib, and W.P. Petersen for enlightening discussions. PdF and AK were supported by the Swiss National Science Foundation. RP and AK were supported by JNICT (Portugal) under grant No. STRDC/C/FAE/1018/93. Numerical simulations for this work were performed on the Intel Paragon and the Cray-Y/MP supercomputers at the ETH.

\section{References}

[1] D. Yu. Grigoriev, V. A. Rubakov, and M. E. Shaposhnikov, Nucl. Phys. B 326737 (1989).

[2] A. I. Bochkarev and G. G. Tsitsishvili, Phys. Rev. D 401378 (1989).

[3] A. I. Bochkarev and Ph. de Forcrand, Phys. Rev. D 44519 (1991).

[4] A. Krasnitz and R. Potting, Phys. Lett. B 318492 (1993); Nucl. Phys. B (Proc. Suppl.) 34613 (1994).

[5] J. Smit and W.H. Tang, Nucl. Phys. B (Proc. Suppl.) 34616 (1994).

[6] D. Yu. Grigoriev, M. E. Shaposhnikov, and N. Turok, Phys. Lett. B 275395 (1992).

[7] I. Montvay, Nucl. Phys. B (Proc. Suppl.) 34631 (1994).

[8] E. Mottola and A. Wipf, Phys. Rev. D 39588 (1989).

[9] M. Dine, O. Lechtenfeld, B. Sakita, W. Fischler, and J. Polchinski, Nucl. Phys. B 342381 (1990).

[10] Since $H(v, a)$ is a periodic function of the $A$ variables, the latter need only be integrated in the range $|a A| \leq \pi$.

[11] G. Bhanot, S. Black, P. Carter, and R. Salvador, Phys. Lett. B 183331 (1987).

[12] M. Creutz and A. Gocksch, Phys. Rev. Lett. 639 (1989). 